\title{
Tolerance-like innate immunity and spleen injury: a novel discovery via the weekly administrations and consecutive injections of PEGylated emulsions
}

This article was published in the following Dove Press journal:

International Journal of Nanomedicine

4 August 2014

Number of times this article has been viewed

\author{
Long Wang* \\ Chunling Wang* \\ Jiao Jiao \\ Yuqing Su \\ Xiaobo Cheng \\ Zhenjun Huang \\ Xinrong Liu \\ Yihui Deng \\ College of Pharmacy, Shenyang \\ Pharmaceutical University, Shenyang, \\ People's Republic of China \\ *These authors contributed equally \\ to this work
}

Correspondence: Yihui Deng

College of Pharmacy, Shenyang

Pharmaceutical University, 103 Wenhua

Road, Shenyang, II0016, People's

Republic of China

Tel +862423986316

Fax +86 2423986316

Email pharmdeng@gmail.com

\begin{abstract}
There has been an increasing interest in the study of the innate immune system in recent years. However, few studies have focused on whether innate immunity can acquire tolerance. Therefore, in this study, we investigated tolerance in the innate immune system via the consecutive weekly and daily injections of emulsions modified with polyethylene glycol (PEG), referred to as PEGylated emulsions (PE). The effects of these injections of PE on pharmacokinetics and biodistribution were studied in normal and macrophage-depleted rats. Additionally, we evaluated the antigenic specificity of immunologic tolerance. Immunologic tolerance against PE developed after 21 days of consecutive daily injections or the fourth week of PE administration. Compared with a single administration, it was observed that the tolerant rats had a lower rate of PE clearance from the blood, which was independent of the stress response. In addition, weekly PE injections caused injury to the spleen. Furthermore, the rats tolerant to PEs with the methoxy group $\left(-\mathrm{OCH}_{3}\right)$ of $\mathrm{PEG}$, failed to respond to the PEs with a different terminal group of PEG or to non-PEG emulsions. Innate immunity tolerance was induced by PE, regardless of the mode of administration. Further study of this mechanism suggested that monocytes play an essential role in the suppression of innate immunity. These findings provide novel insights into the understanding of the innate immune system.
\end{abstract}

Keywords: immunologic tolerance, innate immune system, pharmacokinetics, biodistribution, antigenic specificity

\section{Introduction}

A well-functioning immune system is based on two distinct recognition systems: innate and adaptive. The innate immune system, an evolutionarily ancient component of the host defense, relies on a limited repertoire of receptors to detect invading pathogens, ${ }^{1}$ resulting in a lack of specificity and of "memory". ${ }^{2}$ Providing a rapid but incomplete antimicrobial defense, innate immunity has been considered a separate entity from the adaptive immune response and is regarded as secondarily important in the hierarchy of immune functions. ${ }^{3}$ Nevertheless, Janeway ${ }^{4}$ reported that the adaptive immune response is induced by the exposure of pure antigens combined with adjuvants. This suggests that the innate immune system communicates its biological evaluation of an antigen to the adaptive immune system in some manner. Interest in innate immunity has grown enormously, which has led to intensive studies conducted in many laboratories that seek to integrate these two distinct types of immunity. ${ }^{3}$ A subsequent series of experiments showed that innate immunity plays a role in determining which antigens the acquired immune system responds to and the nature of those responses. ${ }^{5-7}$ The innate and adaptive immune system responses are 
integrated in the vertebrate host into a single immune system, with the innate response preceding, and being necessary for, the adaptive immune response.

The existence of immunological memory in natural killer (NK) cells, which are innate immunes cells, has recently been suggested in a model of chemical hapten-induced contact hypersensitivity. ${ }^{8}$ In addition to B-cells and T-cells, NK cells, can elicit an adaptive immune response in mammals. ${ }^{8,9}$ The novel finding of the adaptive immune features of NK cells indicates that the innate and adaptive immune systems are not only functionally affected but also mutually influence each other. Nevertheless, there is no evidence thus far that T-cell- and B-cell-independent immunologic tolerance can be mediated by innate immunity, likely because of the technical difficulties associated with the experiments or because this question has not drawn enough scientific attention. We sought to more specifically investigate tolerance in innate immunity.

The most direct way to test these novel concepts of immunologic tolerance is to ask the following: how many $\mathrm{T}$ - or B-cells competent enough to react to antigen $\mathrm{X}$ does an individual tolerant to antigen $\mathrm{X}$ possess, compared with non-tolerant controls? ${ }^{10}$ However, research on the tolerance of innate immunity may be hampered by this method because it is not clear which type of cells play a major role in the induction of innate immunologic tolerance. Trying to find cell types using this process is extremely difficult. On the other hand, as the first-line host defense, the innate immune system responds rapidly to limit infection in the initial hours after exposure to microorganisms, ${ }^{11}$ increasing the difficulty in investigating innate immune tolerance. Nonetheless, it is well known that the innate immune system controls the initiation of the adaptive immune response by regulating the expression of co-stimulatory molecules by antigen-presenting cells, and instructs the adaptive immune system to develop a particular effector response. ${ }^{3,12}$ The effector cytokines involved in the adaptive response, in turn, have an effect on the function of the innate response. ${ }^{13}$ We consider these two types of immune responses to be integrated in a closed-loop system in the host. Thus, tolerant innate immunity can be investigated on the basis of adaptive immune tolerance.

The intravenous injected colloidal particles are rapidly recognized by the mononuclear phagocyte system (MPS) and are taken up by macrophages in the liver or spleen. ${ }^{14}$ Therefore, the pharmacokinetics of a single injection dose can be used to evaluate the function of the innate immune system. Nevertheless, the rapid clearance of these conventional carriers results in volatile pharmacokinetic parameters. By contrast, the surface modification of nanocarriers with amphiphilic polyethylene glycol (PEG), termed
PEGylation, ${ }^{15}$ produces a prolonged blood circulation time, which makes PEGylation more suitable for evaluation than the conventional approach. Furthermore, Moghimi and Gray ${ }^{16}$ reported that repeated intravenous administration of poloxamine-coated long-circulating particles at an interval of several days evokes a significant immune response in rats. This phenomenon was named the "accelerated blood clearance (ABC) phenomenon" by Dams et al. ${ }^{17}$ In addition, sequential injections (more than two) of PEGylated nanocarriers led to B-cell anergy in response to cumulative amounts of polymer or encapsulated drugs. ${ }^{18,19}$ In summary, we believe that PEGylated nanocarriers are the best mode to investigate innate immune tolerance.

The lipid emulsion stabilized with emulsifiers such as phospholipids is a promising drug-delivery system. ${ }^{20}$ Lipidemulsion systems meet most requirements for a good parenteral delivery system since they are biodegradable, biocompatible, and physically stable. ${ }^{21}$ Most importantly, lipid emulsions can be prepared on a large industrial scale and are relatively stable below $25^{\circ} \mathrm{C}$ for long periods. ${ }^{22}$ Like other carriers, the lipid emulsions after PEGylation, regarded as a major breakthrough in the application of nanocarriers, have been shown to markedly reduce recognition by the MPS and produce a prolonged blood circulation time when injected intravenously. ${ }^{21}$ In a previous study, we observed that the repeated intravenous administration of PE evoked a significant immune response. ${ }^{23}$ Therefore, in this study, consecutive weekly and daily administrations of PE were used as a tool to investigate innate immune system tolerance. The ratio of the area under the blood concentration-time curve (AUC) $)_{(0-12 \mathrm{~h})}$ in the experimental group compared with that of the control group was selected as an indicator to evaluate the tolerance, due to the high sensitivity of AUC in ANOVA (analysis of variance) tests. ${ }^{24}$

\section{Materials and methods Materials}

Tocopheryl nicotinate (TN) was purchased from Northeast Pharmaceutical Group Co., Ltd (Shenyang, People's Republic of China). Alendronate (AD) was a kind gift from Wansheng Pharmaceutical Co., Ltd (Beijing, People's Republic of China). Injectable soybean lecithin (S75) was obtained from Lipoid GmbH (Ludwigshafen, Germany). Hydrogenated soy phosphatidylcholine (HSPC) was supplied by Lucas Meyer (Düsseldorf, Germany). The medium-chain triglycerides (MCT) were a gift from the Tieling Beiya Medicated Oil Co., Ltd (Tieling, People's Republic of China). Cholesterol was provided by Nanjing Xinbai Pharmaceutical Co., Ltd (Nanjing, People's Republic of China). The distearoylphosphatidyl-N-(methoxy polyoxyethylene 
succinyl) ethanolamine (DSPE-PEG-OCH$H_{3}$ ), distearoylphosphatidyl-N-(3-carboxypropionyl polyoxyethylene succinyl) ethanolamine (DSPE-PEG-COOH), and distearoylphosphatidyl-N-(amine polyoxyethylene succinyl) ethanolamine (DSPE-PEG-NH $\mathrm{N}_{2}$ ) with average molecular weights of PEG $2,000,3,400$, and 3,400, respectively, were provided by Genzyme Corporation (Cambridge, MA, USA). All the other reagents were of chromatographic grade.

\section{Animals}

Male Wistar rats weighing 150-160 g were purchased from the Experimental Animal Center of Shenyang Pharmaceutical University (Shenyang, People's Republic of China). Animal care and experiments were performed in accordance with the guidelines of the local Animal Welfare Committee and of the Principles of Laboratory Animal Care (NIH publication \# 85-23, revised in 1985).

\section{Preparation of emulsions}

TN, MCT, S75 (7.2/30/7, wt/wt/wt), and various groups of DSPE-PEG (S75 and DSPE-PEG, 9/1, molar ratio) were mixed with constant stirring at $55^{\circ} \mathrm{C}$. Sterile water for the injections, heated to $55^{\circ} \mathrm{C}$, was added. The mixture was immediately and quickly stirred, and incubated at $55^{\circ} \mathrm{C}$ for 10 minutes to produce the prime emulsion. The resulting emulsion was sonicated using a laboratory ultrasonic cell pulverizer (JY92-II; Ningbo Scientz Biotechnology Co., Ltd, Zhejiang, People's Republic of China) for at least a 2-minute cycle (200 W) and an additional 6-minute cycle $(400 \mathrm{~W})$. The obtained emulsions were sized by extrusion through polycarbonate membrane filters with pore sizes of 0.22 $\mu \mathrm{m}$ at $25^{\circ} \mathrm{C}$ and were adjusted to an isotonic level with the injection of $50 \%$ glucose. The mean diameters and zeta potentials of the emulsions were determined in purified water at $23^{\circ} \mathrm{C}$ using the submicron particle analyzer (Nicomp 380M; Particle Sizing Systems, Inc., Santa Barbara, CA, USA). The mean particle size of the PEGylated emulsions (PEs) was 124.9 $\pm 3.2 \mathrm{~nm}(\mathrm{n}=6)$, and the zeta potential was $-38.3 \pm 2.6 \mathrm{mV}(\mathrm{n}=6)$.

\section{Pharmacokinetics and biodistribution of the PEs}

Male Wistar rats were randomly divided into four groups: those receiving 1) weekly administration of $\mathrm{PE}, 2$ ) daily injections of PE, 3) 5\% glucose injection ( $5 \% \mathrm{Glu})$, and 4) the non-treated control group not receiving any treatment. The rats receiving daily injections of $\mathrm{PE}$ were injected with the PEs without TN at a dose of $5 \mu \mathrm{mol}$ phospholipids $/ \mathrm{kg}$. Three rats were killed on days 7, 11, 14, 17, and 21 after the respective injections. The rats receiving weekly administrations of PE were treated with PEs without TN at the same dose, $5 \mu \mathrm{mol}$ phospholipids/kg; the rats in the $5 \%$ Glu group were given only an injection of glucose; and the control group did not receive any treatment. For each injection, three randomly selected rats from each group received the same dose of TN-labeled PEs to investigate the pharmacokinetics and biodistribution of PE. The injection schemes are presented in Figure 1. All the administrations were intravenously injected into the tail vein. At $0.0167,0.083,0.25,0.5,1.0$, 4.0, 8.0, and 12.0 hours after the intravenous injections, blood samples were obtained via eye punctures, and the samples were centrifuged at $1,078 \times g$ for 10 minutes to separate the plasma. After obtaining the last blood sample at 12 hours, the livers and spleens were excised and rinsed in ice-cold normal saline. The blood samples and tissue samples were stored at $-20^{\circ} \mathrm{C}$ for future use.

The concentration of TN in the plasma and tissue samples was analyzed by high performance liquid chromatography (HPLC) using a P230 pump and a UV230 ultraviolet-visible spectroscopy Detector (Da Lian Elite Analytical Instruments Co., Ltd, Liaoning, People's Republic of China) and separated using a Hypersil ${ }^{\circledR}$ BDS C18 column (200 mm $\times 4.6$ $\mathrm{mm}$ ) containing particles measuring $5 \mu \mathrm{m}$ in diameter at $30^{\circ} \mathrm{C}$. The ultraviolet wavelength was $264 \mathrm{~nm}$. The mobile phase consisted of methanol/isopropanol $(80 / 20, \mathrm{v} / \mathrm{v})$ at a flow rate of $1 \mathrm{~mL} /$ minute. Before the analysis, the plasma samples and tissue samples were treated as follows: $100 \mu \mathrm{L}$ of the plasma samples or homogenates (equivalent to 0.1 $\mathrm{g}$ tissue) were mixed with methanol $(100 \mu \mathrm{L})$, an internal standard $(100 \mu \mathrm{L})$, tocopheryl acetate $(100 \mu \mathrm{g} / \mathrm{mL})$, and n-hexane $(600 \mu \mathrm{L})$. The entire mixture was vortexed for 5 minutes and centrifuged at $10,000 \mathrm{rpm}$ for 10 minutes. The supernatant $(500 \mu \mathrm{L})$ was dried using a CentriVap ${ }^{\circledR}$ Centrifugal Vacuum Concentrator (Labconco Corporation, Kansas City, MO, USA) and dissolved in the mobile phase $(100 \mu \mathrm{L})$. The resulting mixture was vortexed for 1 minute and centrifuged at 10,000 rpm for 10 minutes. The supernatant $(20 \mu \mathrm{L})$ was collected and used for the HPLC analysis. The in vitro release of TN from the PEGylated emulsions was also investigated and the result was shown in Figure S1 (see Supplementary materials).

\section{Histopathology}

The rats injected with the intravenous administrations of PE at a dose of $5 \mu \mathrm{mol}$ phospholipids $/ \mathrm{kg}$ with no prior treatment were killed on the indicated day, and their spleens were removed. The spleens were fixed in $20 \%$ neutral buffered formalin, embedded in paraffin wax, sectioned at 4 or $5 \mu \mathrm{m}$, and stained with hematoxylin and eosin. The pathological sections were observed by microscopy. 
A

Consecutive injections

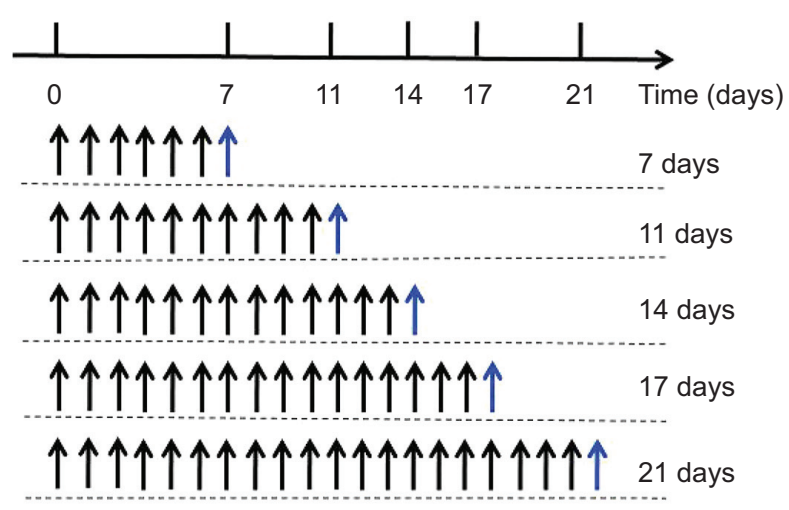

B

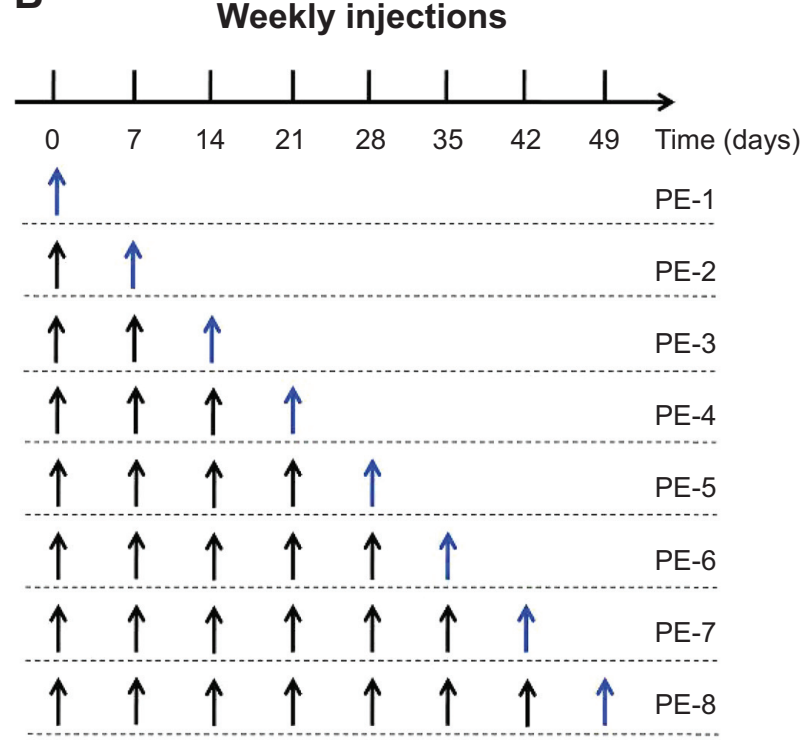

Figure I Time schedule for the experiment.

Notes: (A) The consecutive injections of PE group, $n$ days represent the nth-day PE injection. (B) The weekly injections of PE group, PE-n represents the nth-week PE injection. Black arrows indicate the injection of the blank PE, and blue arrows indicate the injection of PE.

Abbreviations: PE, PEGylated emulsion; PEG, polyethylene glycol.

\section{Macrophage depletion experiments}

To study the effect of macrophages on the induction of innate immunity suppression, tissue macrophages were depleted by intravenous injection of unsized multilamellar HSPCcholesterol-liposomes containing AD (AD-L). AD-L was injected 48 hours before the administration of TN-labeled $\mathrm{PE}$, with an $\mathrm{AD}$ dose of $3 \mathrm{mg} / \mathrm{kg}$ in the normal and tolerant rats. Injection of liposomes without $\mathrm{AD}$ was used as the control. The interval of 48 hours was chosen because macrophage depletion in the liver and spleen is maximal at this time point. ${ }^{25}$

\section{Statistical analysis}

The data are presented as the mean \pm standard deviation. The statistical analysis was performed using Student's $t$-test with SPSS 16.0 (SPSS Inc., Chicago, IL, USA) software. $P<0.05$ was considered statistically significant.

\section{Results}

\section{The effect of weekly PE administrations} and daily PE injections on the pharmacokinetics and biodistribution of PEs

\section{Effect of weekly PE administrations}

Weekly injections of PE at a dose of $5 \mu \mathrm{mol}$ phospholipids $/ \mathrm{kg}$ dramatically influenced the circulatory half-life of the successively administered PE in the rats. The results shown in
Figure 2 demonstrate that the blood clearance of the second dose was sharply accelerated (AUC ratio $=0.10 \pm 0.06$ ). Following successive injections, the $\mathrm{ABC}$ phenomenon became less pronounced and the blood concentration normalized, but PE accumulation in the liver remained slightly high at the fifth injection (4 weeks after the first injection). The $\mathrm{AUC}_{(0-12 \mathrm{~h})}$ level of the fifth, sixth, and seventh injections of PE was, respectively, 1.37, 1.39, and 1.46 times greater than that at the first injection. A significantly increased accumulation of $\mathrm{PE}$ in the spleen was observed at the seventh injection $(P<0.001)$.

\section{Effect of daily PE injections}

The influence of consecutive daily injections on the pharmacokinetics and biodistribution of PE was studied. As shown in Figure 3, blood clearance and hepatic and splenic accumulation significantly increased after 7 days of sequential injection (AUC ratio $=0.10 \pm 0.01$ ). An inverse correlation between the dose frequency and the magnitude of the $\mathrm{ABC}$ phenomenon was observed. The $\mathrm{ABC}$ phenomenon was avoided after 21 days of consecutive administrations. Interestingly, the blood elimination rate of PE injected after 21 days was less than a single dose (AUC ratio $=1.37 \pm 0.11, P<0.05$ ).

\section{The antigenic specificity of immunologic tolerance}

The most important characteristic of immunologic tolerance is antigen-specificity. The host forms immunologic tolerance to an antigen, but the individual can still mount an immune response 
A

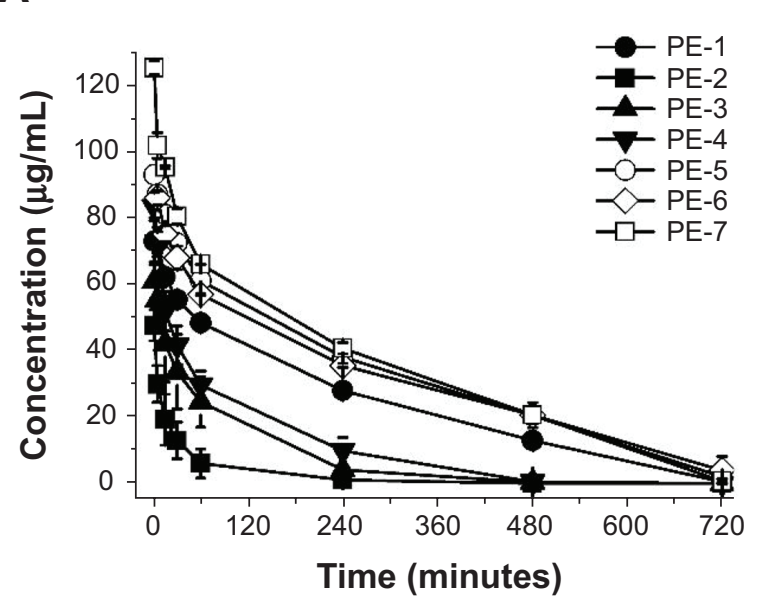

B

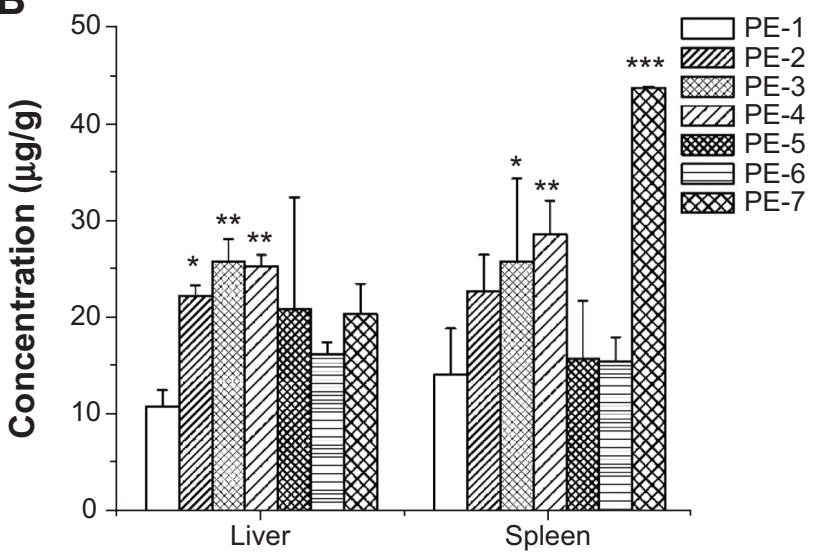

Figure 2 The effect of weekly injections on the pharmacokinetics and biodistribution of PE.

Notes: (A) Blood clearance. (B) Hepatic and splenic accumulation 12 hours after intravenous injection of the test dose. PE-n represents the nth-week PE injection. Data are shown as mean $\pm \mathrm{SD} ; \mathrm{n}=3$. P-values apply to differences between the control and treated rats; $* P<0.05$, $* * P<0.01$, $* * * P<0.00 \mathrm{I}$.

Abbreviations: PE, PEGylated emulsion; PEG, polyethylene glycol; SD, standard deviation.

to other antigens. In this study, immunologic tolerance was induced by successive injections of the PEs, and it was possibly specific to the terminal group of PEG or to PEG.

\section{Antigenic specificity to the terminal group of PEG}

To investigate the antigenic specificity to the terminal group of PEG, the tolerant rats, which were immunized with PEs with the methoxy group of PEG $\left(\mathrm{PE}-\mathrm{OCH}_{3}\right)$, were injected with PEs with the amino group (PE-NH ${ }_{2}$ ) and carboxyl group (PE$\mathrm{COOH})$ of PEG. As shown in Figure 4, the plasma concentration of $\mathrm{PE}-\mathrm{NH}_{2}$ and $\mathrm{PE}-\mathrm{COOH}$ in the $\mathrm{PE}$ group increased correspondingly, especially within 1 hour. The $\mathrm{AUC}_{(0-1 \mathrm{~h})}$ of $\mathrm{PE}-\mathrm{NH}_{2}$ and $\mathrm{PE}-\mathrm{COOH}$ administered in the normal rats, the 5\% Glu group, and the PE group was 10.40, 15.42, and 34.18 , and $40.51,37.10$, and $53.47 \mathrm{mg} / \mathrm{L} \cdot$ hour, respectively. The results demonstrate that $\mathrm{PE}-\mathrm{OCH}_{3}$-tolerant rats failed to respond to $\mathrm{PE}-\mathrm{NH}_{2}$ and $\mathrm{PE}-\mathrm{COOH}$. A large increase in splenic accumulation was observed in the tolerant rats that received PE-NH $_{2}(P<0.05)$. There were no remarkable differences in rats that received weekly injections of $5 \%$ Glu.

\section{Antigenic specificity to PEG}

The tolerant rats were given non-PEs and conventional emulsions (CEs) to further determine antigenic specificity to PEG. From Figure 5, it is apparent that the clearance of CE in the PE group and the 5\% Glu group decreased 1.48- and
A

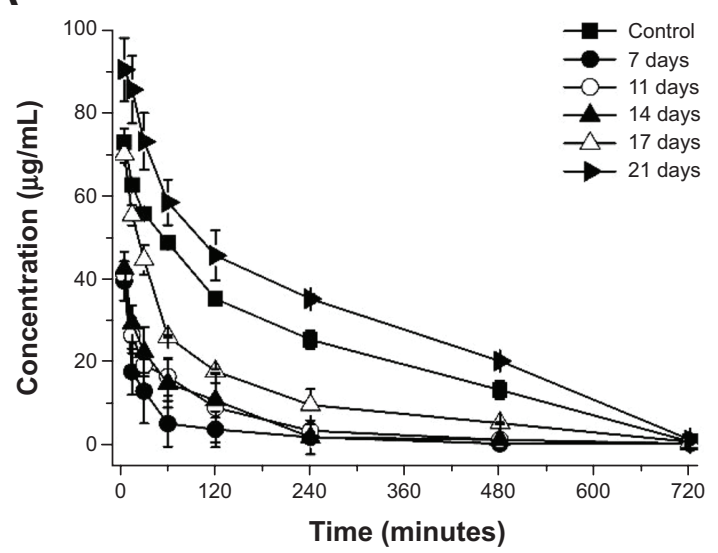

B

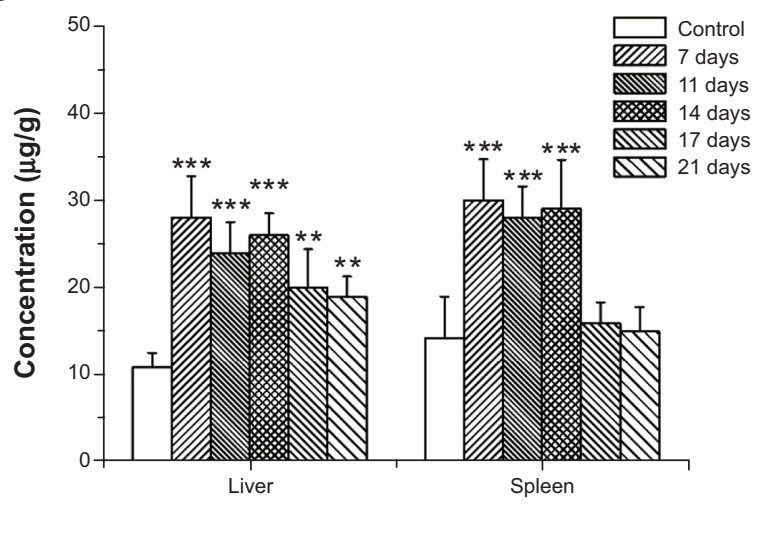

Figure 3 The effect of daily injections on the pharmacokinetics and biodistribution of PE.

Notes: (A) Blood clearance. (B) Hepatic and splenic accumulation 12 hours after intravenous injection of the test dose. $\mathrm{n}$ days represent the nth-day PE injection. Data are shown as mean $\pm \mathrm{SD}, \mathrm{n}=3$. $P$-values apply to differences between the control and treated rats; $* * P<0.0 \mathrm{I}, * * * P<0.001$.

Abbreviations: PE, PEGylated emulsion; PEG, polyethylene glycol; SD, standard deviation. 
A1

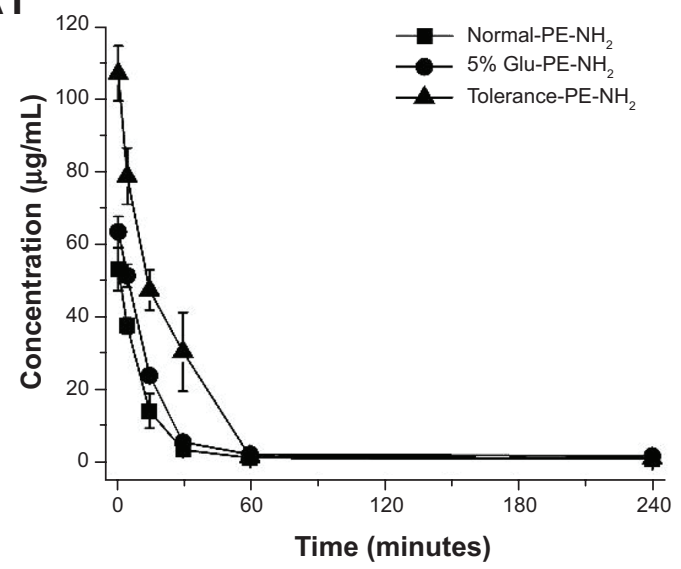

A2

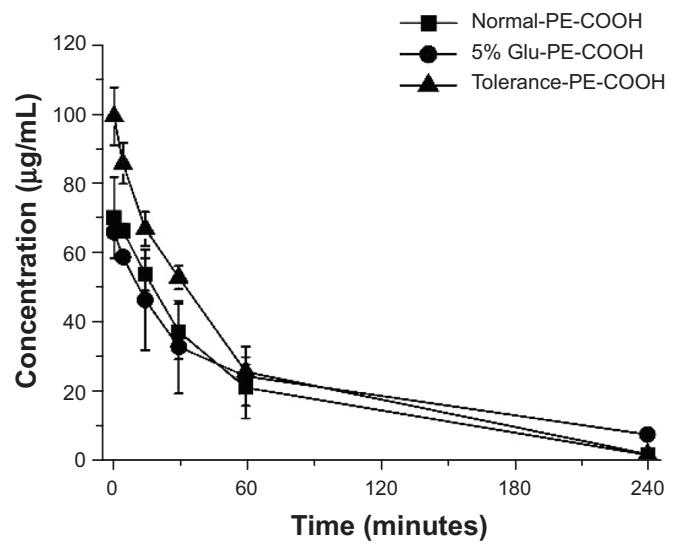

B1

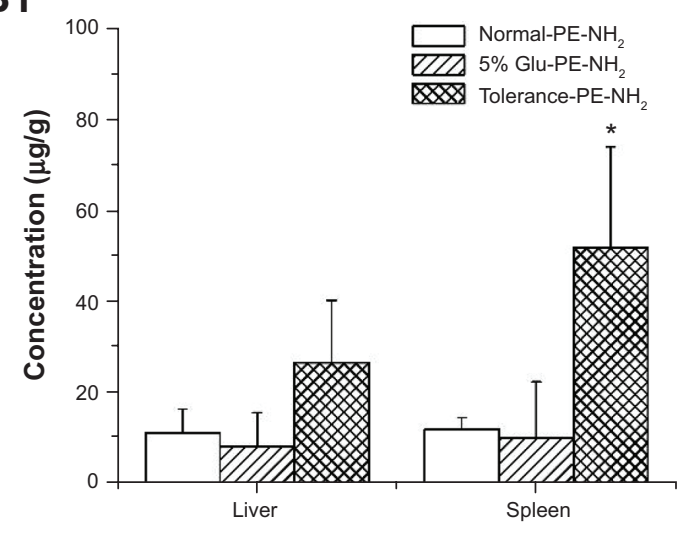

B2

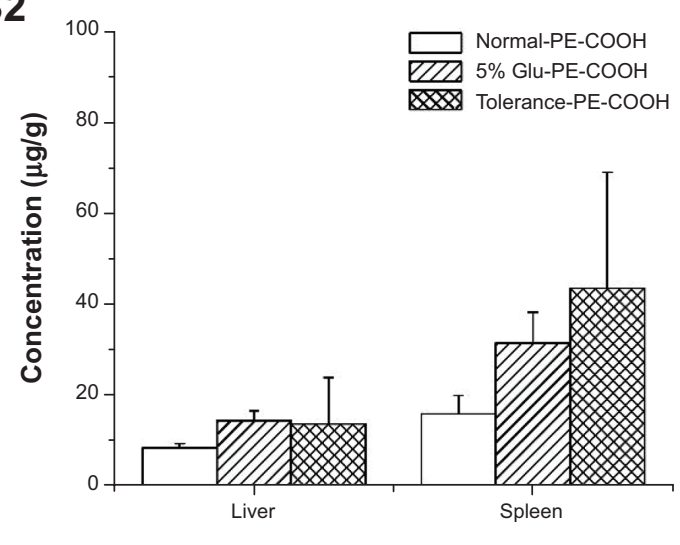

Figure 4 The antigenic specificity to terminal group of PEG in the tolerant rats induced by $\mathrm{PE}-\mathrm{OCH}_{3}$.

Notes: (AI and A2) Blood clearance. (BI and B2) Hepatic and splenic accumulation I2 hours after intravenous injection of the test dose. (AI and B I) Intravenous injection of $\mathrm{PE}-\mathrm{NH}_{2}$. (A2 and $\mathbf{B} 2$ ) Intravenous injection of $\mathrm{PE}-\mathrm{COOH}$. Data are shown as mean $\pm \mathrm{SD}, \mathrm{n}=3 ; * \mathrm{P}<0.05$.

Abbreviations: PE, PEGylated emulsion; PEG, polyethylene glycol; SD, standard deviation; PE-OCH, PEGylated emulsions modified with the methoxy group of DSPEPEG; PE-NH $\mathrm{N}_{2}$, PEGylated emulsions modified with the amino group of DSPE-PEG; PE-COOH, PEGylated emulsions modified with the carboxyl group of DSPE-PEG; DSPE, I,2-distearoyl-sn-glycero-3-phosphoethanolamine.

1.15-fold, respectively, compared with those rats that received a single injection dose.

\section{Spleen injury}

The spleen weight of the rats decreased with increasing frequency of PE injections. The spleen index was decreased from $3.506 \pm 0.231 \mathrm{mg} / \mathrm{g}$ to $1.228 \pm 0.176 \mathrm{mg} / \mathrm{g}$. In this study, we thought that the changes in spleen weight would be related to the weekly injections of PE and would be confirmed with histopathological evaluation. It is shown in Figure 6 that in comparison with the normal rats, the proportion of white pulp was decreased and red pulp was increased. The PE group follicles were markedly reduced in size and poorly demarcated from the marginal zone and the lymphoid sheath.

\section{Involvement of macrophages in the induction of innate immune tolerance}

To study the involvement of macrophages in the induction of innate immune tolerance, hepatic and splenic macrophages were depleted by intravenous injection of AD-L in the normal and tolerant rats.

\section{Macrophage depletion in normal rats}

PE was injected in the normal rats that had received AD-L 48 hours before. As shown in Figure 7, compared with a single injection of $\mathrm{PE}$ in non-depletion rats, the blood clearance of PE significantly decreased (AUC ratio $=2.25 \pm 0.32$ ). There was enhanced accumulation in the spleen $(P<0.001)$ and slightly decreased accumulation in the liver.

\section{Macrophage depletion in tolerant rats}

The tolerant rats were intravenously injected with a single dose of AD-L, and 48 hours after this initial injection, the second dose of PE was administered. As described in Figure 8, the $\mathrm{AUC}_{(0-12 \mathrm{~h})}$ of the PE administered in the normal rats, tolerant rats, and tolerant rats with macrophage depletion was $257.5,477.6$, and $601.8 \mathrm{mg} / \mathrm{L} \cdot$ hour, respectively. Regardless of macrophage depletion, the tolerant rats had significantly 


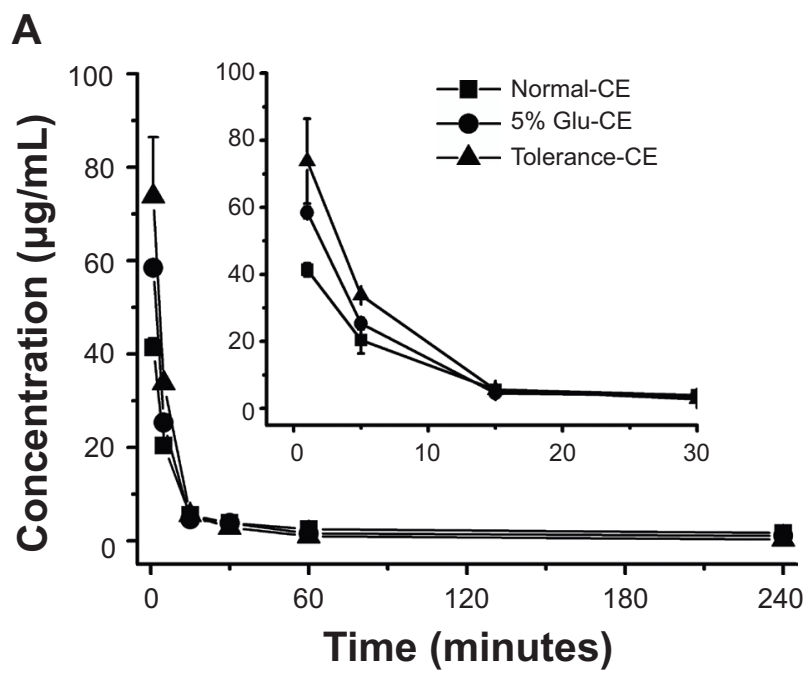

B

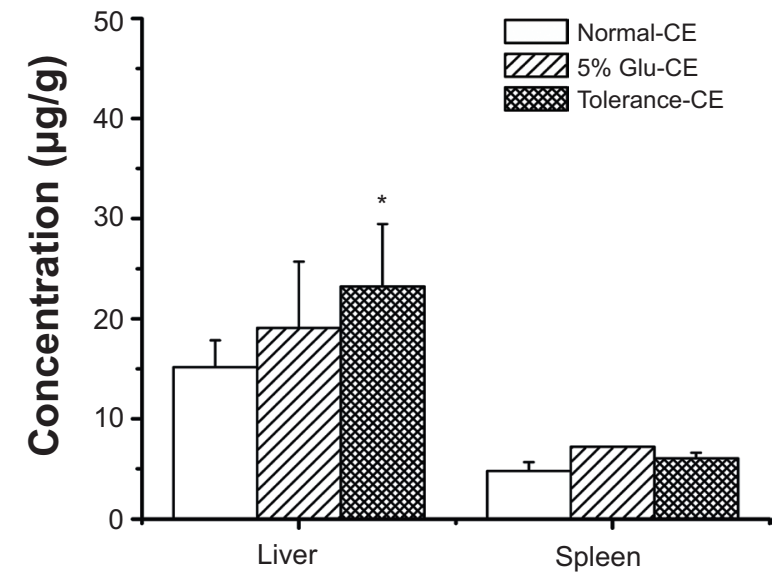

Figure 5 The antigenic specificity to PEG in the tolerant rats induced by $\mathrm{PE}-\mathrm{OCH}_{3}$.

Notes: (A) Blood clearance. The inset shows a magnified view of 0 to 30 minutes of blood clearance. (B) Hepatic and splenic accumulation 12 hours after intravenous injection of the test dose. Data are shown as mean $\pm S D, n=3, * P<0.05$.

Abbreviations: $\mathrm{CE}$, conventional emulsion; PE, PEGylated emulsion; PEG, polyethylene glycol; SD, standard deviation; Glu, glucose injection; PE-OCH ${ }_{3}$, PEGylated emulsions modified with the methoxy group of DSPE-PEG; DSPE, I,2-distearoyl-sn-glycero-3-phosphoethanolamine.

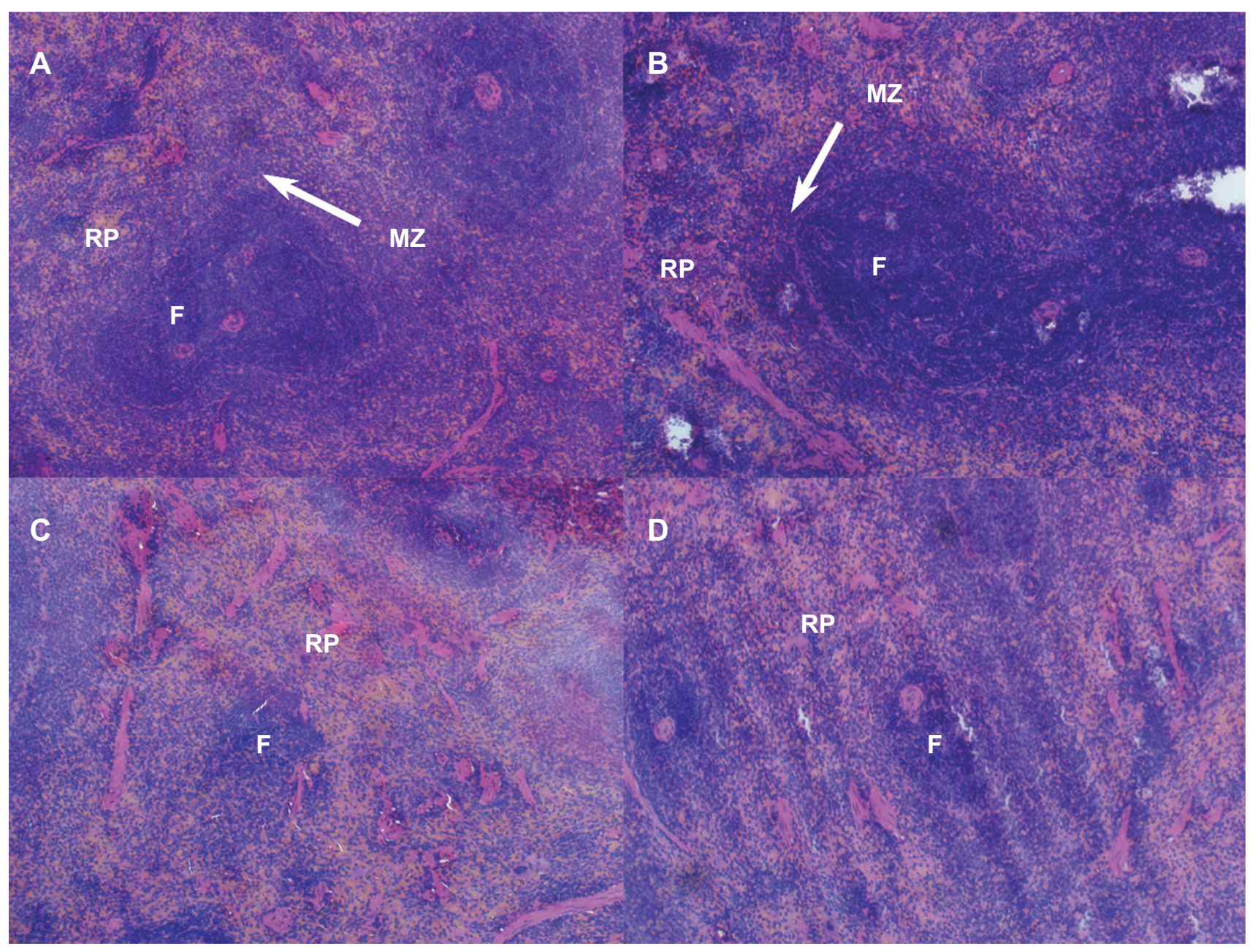

Figure 6 The pathological photos of spleen.

Notes: Magnification $\times 20$. (A) The control group. (B) The $5 \%$ Glu group. (C and D) The PE group. (C) Represents that the proportion of white pulp is decreased and red pulp is increased. (D) Represents that follicles are markedly reduced in size and poorly demarcated from the marginal zone and the lymphoid sheath. Abbreviations: F, follicle; MZ, marginal zone; RP, red pulp; Glu, glucose injection; PE, PEGylated emulsion. 
A

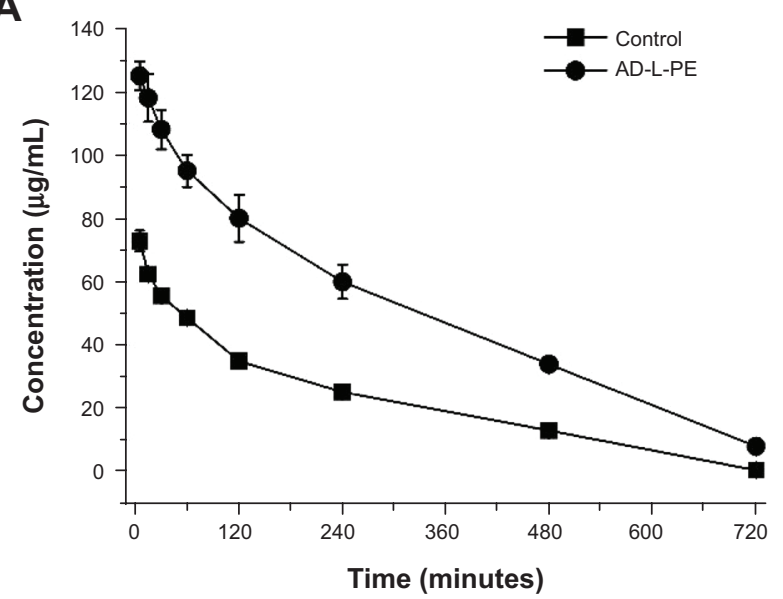

B

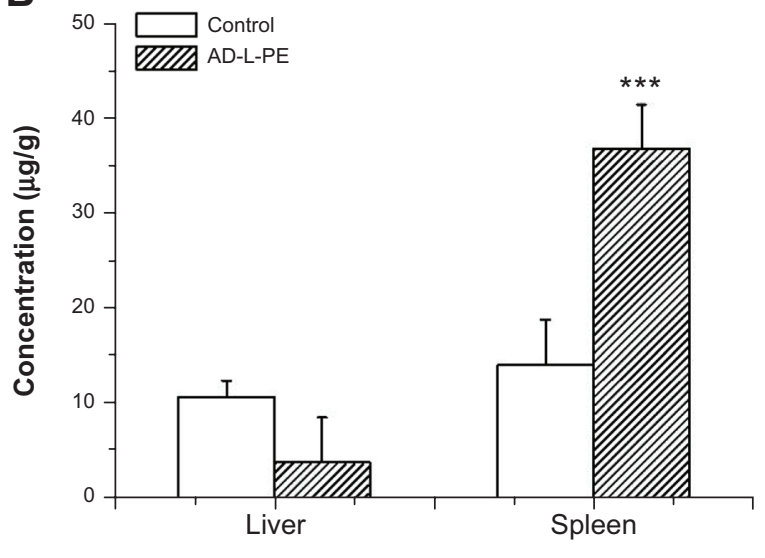

Figure 7 The effect of macrophage depletion in the normal rats on the pharmacokinetics and biodistribution of PE.

Notes: (A) Blood clearance. (B) Hepatic and splenic accumulation 12 hours after intravenous injection of the test dose. Data are shown as mean $\pm \mathrm{SD}, \mathrm{n}=3$. $* * * P<0.00 \mathrm{I}$.

Abbreviations: AD-L, liposomes containing alendronate; PE, PEGylated emulsion; PEG, polyethylene glycol; SD, standard deviation.

increased splenic accumulation of PE. There was no change in hepatic accumulation of PE in the tolerant rats, and a slight decrease in macrophage-depleted rats.

\section{Discussion}

The results described in this paper show that the first dose of PE caused a remarkable reduction in PE circulation time and an increase in the hepatic and splenic accumulation of the second dose given at a 7-day interval, reproducing what has been called, the $\mathrm{ABC}$ phenomenon. In this phenomenon, repeated administration of PEGylated colloidal particles at an interval of several days evokes a significant immune response in rats. The rats failed to respond to the PE after being given four doses at 7-day weekly intervals, suggesting that the rats established immunologic tolerance due to excessive stimulation of B-cells (Figure 2). The immunologic tolerance was also induced by 21 days of consecutive daily injections of PE (Figure 3). Interestingly, compared with a single injection, the decreased blood clearance of $\mathrm{PE}$ was observed in the rats receiving weekly PE administrations after the fifth to seventh doses and in the sequential injections after 21 days (Figures 2 and 3). The AUC ratio of the two groups was greater than 1 (Table 1). The results suggest that multiple injections of PE could result in a suppression of the innate immune system. This inhibition was independent of the mode of administration.

With the attempt to further clarify the effect of repeated administrations of PE on the immune system, we investigated
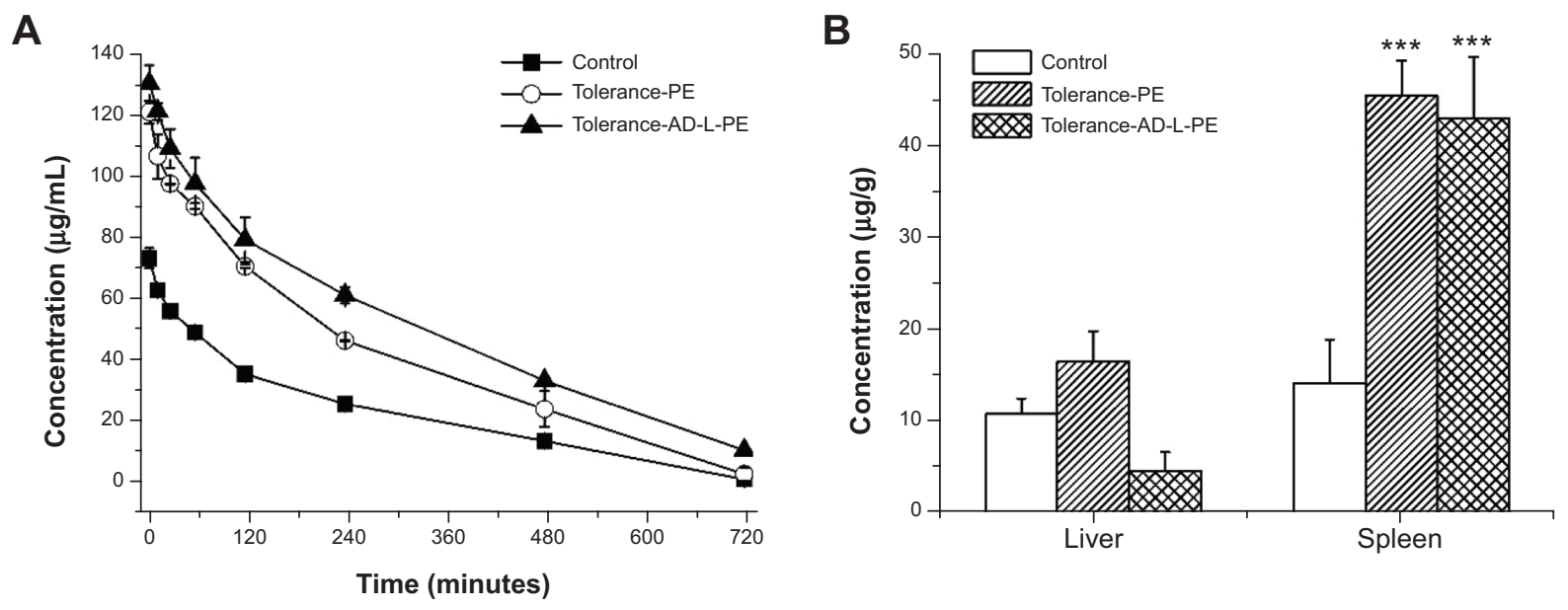

Figure 8 The effect of macrophage depletion in the tolerant rats on the pharmacokinetics and biodistribution of PE.

Notes: (A) Blood clearance. (B) Hepatic and splenic accumulation 12 hours after intravenous injection of the test dose. Data are shown as mean \pm SD, $\mathrm{n}=3$; $* * * P<0.00 \mathrm{I}$. Abbreviations: AD-L, liposomes containing alendronate; PE, PEGylated emulsion; PEG, polyethylene glycol; SD, standard deviation. 
Table I The AUC ratio of weekly administrations and daily injections of PE

\begin{tabular}{llll}
\hline $\begin{array}{l}\text { Weekly } \\
\text { injections }\end{array}$ & $\begin{array}{l}\text { AUC } \\
\text { ratio }\end{array}$ & $\begin{array}{l}\text { Daily } \\
\text { injections }\end{array}$ & $\begin{array}{l}\text { AUC } \\
\text { ratio }\end{array}$ \\
\hline PE-2 & $0.10 \pm 0.06 * * *, d$ & $7 \mathrm{~d}-\mathrm{PE}$ & $0.10 \pm 0.01 * * *$ \\
PE-3 & $0.32 \pm 0.09 * * *$ & $1 \mathrm{I} \mathrm{d}-\mathrm{PE}$ & $0.21 \pm 0.08 * * *$ \\
PE-4 & $0.45 \pm 0.09 * *$ & $14 \mathrm{~d}-\mathrm{PE}$ & $0.21 \pm 0.05 * * *$ \\
PE-5 & $1.37 \pm 0.08 *$ & $17 \mathrm{~d}-\mathrm{PE}$ & $0.50 \pm 0.18 * *$ \\
PE-6 & $1.39 \pm 0.09 *$ & $21 \mathrm{~d}-\mathrm{PE}$ & $1.37 \pm 0.1 \mathrm{I}^{*}$ \\
PE-7 & $1.46 \pm 0.28 *$ & & \\
\hline
\end{tabular}

Notes: ${ }^{\text {TTh }} \mathrm{AUC}$ ratio was calculated as follows: $\mathrm{AUC}$ ratio $=\mathrm{AUC}_{(0-12 \mathrm{~h})}$ of the treated group/AUC $\mathrm{C}_{(0-12 \mathrm{~h})}$ of the control group; 'PE-n represents the nth-week $\mathrm{PE}$ injection; ${ }^{c} n$ d-PE represents the nth-day $P E$ injection; ${ }^{d} P$-values apply to differences between the treated groups and I; $* P<0.05, * * P<0.0$ I, $* * * P<0.001$.

Abbreviations: AUC, area under the blood concentration-time curve; PE, PEGylated emulsion; PEG, polyethylene glycol. the role of the stress response. Selye ${ }^{26}$ demonstrated that there is a rapid decrease in the size of the thymus, spleen, and lymph glands when rats are exposed to cold, surgical injury, the production of spinal shock, and other stressors; they further proposed that the stress response has certain effects on the immune system. Moreover, Weiss et $\mathrm{al}^{27}$ showed that the immune response is suppressed by slight stress, enhanced by moderate stress, and significantly inhibited by high stress. In this study, continuous intravenous injections and blood collection from the orbital venous plexus kept the experimental animals in a slightly stressed state. Our results showed that the blood clearance of $\mathrm{PE}$ in the $5 \%$ Glu group was similar to the control group ( $\mathrm{AUC}$ ratio $=1.03 \pm 0.05$ ), and the $\mathrm{AUC}_{(0-12 \mathrm{~h})}$

\section{Monocytes}

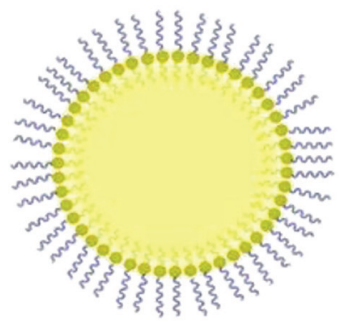

PEGylated emulsions

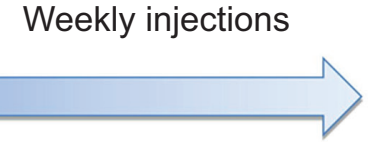

Daily injections

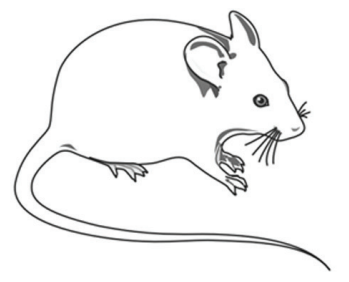

Tolerant rats
No antigenic specificity

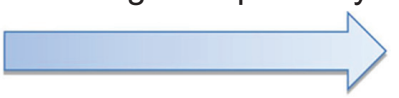

Continuous intravenous injections

$>$ Blood draws from the orbital venous plexus

$>$ Spleen injury

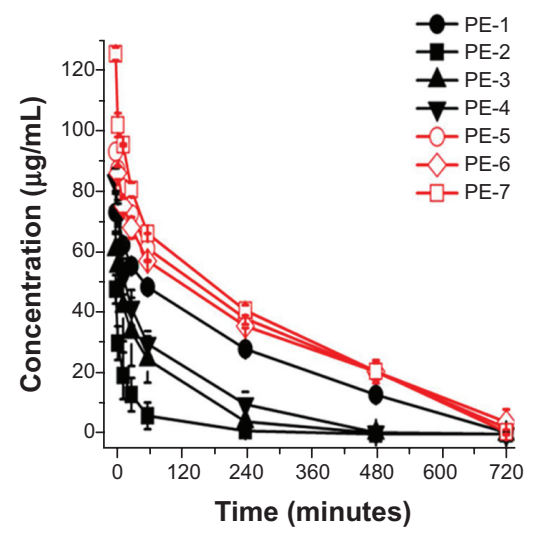

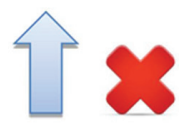

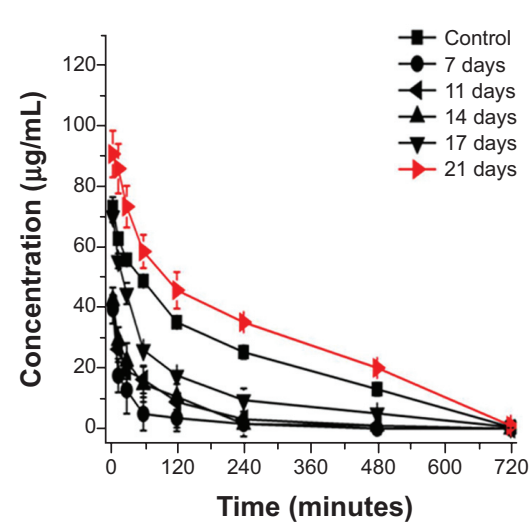

Innate immune tolerance

Figure 9 The summary of the findings and possible mechanisms associated with innate immune tolerance with PE. Note: PE-n represents the nth-week PE injection, $n$ days represent the nth-day PE injection.

Abbreviations: PE, PEGylated emulsion; PEG, polyethylene glycol. 
after the fifth PE injection with or without obtaining blood was $384.4 \pm 5.8$ and $376.2 \pm 3.2 \mathrm{mg} / \mathrm{L} \cdot$ hour, respectively (data not shown). It indicated that continuous intravenous injections and collection of blood from the orbital venous plexus had no effect on the decreased clearance of PE. It is tempting to speculate that the repeated injection of PE contributed to the suppression of the innate immunity.

To further confirm the type of immunologic tolerance, we studied the antigen-specificity of immunologic tolerance induced by weekly PE injections. Antigen-specific tolerance is achieved when the host forms an immunologic tolerance to an antigen and fails to respond to the same antigen after future exposure. However, the individual can still induce an immune response to other antigens. It has been reported that PEGylated nanocarriers used in a first injection, regarded as TI-2 (T-cell independent type 2) antigens, stimulate the splenic marginal zone $\mathrm{B}$ to produce anti-PEG immunoglobulin (Ig), resulting in the $\mathrm{ABC}$ of subsequently administered nanocarriers. ${ }^{28,29}$ Cheng et $\mathrm{al}^{30,31}$ had demonstrated that anti-PEG antibody (IgM) obtained following immunization with PEGylated $\beta$-glucuronide recognizes the repeating $-\left(\mathrm{O}-\mathrm{CH}_{2}-\mathrm{CH}_{2}\right) n-$ subunit of PEG. This raises the possibility that the repeating subunit may be an immunogenic epitope of PEG and a binding site for the derived anti-PEG IgM. Therefore, the immunologic tolerance induced by weekly injections of PE was likely to be specific to the terminal group of PEG or to PEG. Nevertheless, we observed that the AUC ratio of $\mathrm{PE}-\mathrm{NH}_{2}, \mathrm{PE}-\mathrm{COOH}$, and $\mathrm{CE}$ administered in the tolerant rats was $3.29,1.32$, and 1.46 , respectively (Figures 4 and 5). In other words, the $\mathrm{PE}-\mathrm{OCH}_{3}$-tolerant rats did not respond to $\mathrm{PE}-\mathrm{NH}_{2}, \mathrm{PE}-\mathrm{COOH}$, or the $\mathrm{CE}$. The results indicate that immunologic tolerance without antigen-specificity was evoked by weekly administrations of $\mathrm{PE}-\mathrm{OCH}_{3}$, and they further support that innate immune function was suppressed.

The spleen is responsible for initiating innate and adaptive immune responses to antigens and pathogens, and is considered an important organ in evaluating immune system function. ${ }^{32}$ In the present study, significant changes in spleen weight were observed in the PE group compared with the 5\% Glu group, with a considerably increased concentration of TN in the spleen of tolerant rats (Figures 2 and 4). In addition, spleen injury, characterized by a reduction in the size of the follicles and the disappearance of the marginal zone, was induced by weekly injections of PE (Figure 6). However, Ishida et $\mathrm{al}^{33}$ reported that in the splenectomized rats, a single dose of PEGylated liposomes was eliminated faster and showed higher liver accumulation than that seen in the control rats. Based on this study, we consider it conceivable that the suppression of innate immunity was not evoked by spleen injury.

It is well known that the blood clearance of intravenously injected colloidal particles depends on the immune cells of MPS. ${ }^{34,35}$ The determination of blood cells was employed to investigate the effect of immune cells in the peripheral blood on the clearance of PE. Blood samples were collected immediately after the intravenous injection of PE, and the increased percentage of monocytes, leukocytes, lymphocytes, and neutrophilic granulocytes were 467\%, 326\%, $320 \%$, and $306 \%$, respectively (data not shown). The results demonstrate that blood monocytes were mainly responsible for the clearance of PE. Moreover, Laverman et $\mathrm{al}^{36}$ studied the involvement of macrophages in the induction and the effectuation of the enhanced clearance effect occurring after the second injection of PEG-liposomes. When macrophages were depleted before the first and second injections, PEGliposomes had a normal long circulation time after the second injection. This reveals that macrophages are involved in the production of the ABC phenomenon. In addition, it was also clear that the splenectomized rat failed to completely reverse the rapid clearance and to reverse the increased hepatic accumulation of PEGylated liposomes to control levels. ${ }^{33}$ This suggests that in addition to the spleen, another serum factor(s) or tissue(s) was involved in this phenomenon. Ishida et $\mathrm{a}^{33}$ and Ichihara et $\mathrm{al}^{37}$ speculated that macrophages, including Kupffer cells, acquire the ability to recognize and aggressively take up "invisible" PEGylated liposomes, without the involvement of any opsonizing factor(s). Based on the above discussion, macrophages seem to be responsible for the induction of the $\mathrm{ABC}$ phenomenon. The data, as shown in Figures 2 and 3, indicate that repeated injections of PE can evoke a remarkable $\mathrm{ABC}$ phenomenon. We speculate that the consecutive daily and weekly PE administrations might have had an effect on the function of monocytes and macrophages. Figure 7 shows that macrophage depletion caused a remarkable reduction in the clearance of PE in the normal rats, indicating that macrophages were involved in the blood removal of PE. These data are consistent with the blood clearance data. Furthermore, enhanced accumulation in the spleen $(P<0.001)$ and decreased accumulation in the liver $(P<0.05)$ were also observed, indicating that the Kupffer cells were depleted by intravenous injection of liposomes containing $\mathrm{AD}$, but not macrophages in the spleen. Moreover, compared with the tolerant rats, increased AUC and decreased PE accumulation in the liver were observed in the tolerant rats with macrophage depletion. These results 
suggest that the Kupffer cells did not play a major role in this process. On the other hand, it has been reported that the function of blood monocytes is inhibited by intravenous injection of AD-loaded nanoparticles. ${ }^{38,39}$ Therefore, we propose that monocytes may play a role in inducing innate immunity tolerance upon consecutive daily injections and weekly administrations of PEs, but the detailed mechanism requires further investigation.

In the present study, we first proposed that innate immunity tolerance was induced by the consecutive injections and weekly administrations of PE in rats (Figure 9). This generalized tolerance feature of the innate immune system is defined as decreased recognition and clearance of invading pathogens, even of the infectious and inflammatory diseases. The results reported here provide a novel insight into the understanding of the relationship between innate and adaptive immune systems and further supplement the classical theory of immunology. Furthermore, our results have potential implications for the clinical application of PEs. The unexpected inhibition of the innate immune system was undesirable. The induced innate immunity tolerance has a marked effect on PE, even conventional fat emulsions in clinical situations, and the decreased blood clearance of the drug formulations can compromise their safety and therapeutic efficacy. In addition, if emulsions contain toxic drugs with a small therapeutic window, the increased concentration in the blood could cause adverse systemic toxicity. The immunologic tolerance was a key mechanism for maintaining the stability of the body based on antigenspecific and immune memory. The possible suppression of the innate immunity function led to a risk of infection, which is a negative effect for the treatment of diseases. The dysfunction of the immune system was the direct cause of the induced infectious disease or tumors. ${ }^{40,41}$ These findings may provide new perspectives regarding our understanding of the clinical application of PE.

\section{Conclusion}

To our knowledge, this study is the first to report that tolerance in innate immunity was induced by multiple injections of PEs in rats. Our results reveal that monocytes may lead to the suppression of the innate immune system. These findings may provide new perspectives in our conventional understanding of the innate immune system.

\section{Acknowledgment}

The National Natural Science Foundation of China supported this study (Grant No 81072602).

\section{Disclosure}

The authors report no conflicts of interest in this work.

\section{References}

1. Beutler B. Innate immunity: an overview. Mol Immunol. 2004;40(12): 845-859.

2. Fearon DT. Seeking wisdom in innate immunity. Nature. 1997;388(6640): 323-324.

3. Medzhitov R, Janeway CA Jr. Innate immunity: impact on the adaptive immune response. Curr Opin Immunol. 1997;9(1):4-9.

4. Janeway CA. Approaching the asymptote? Evolution and revolution in immunology. Paper presented at: Cold Spring Harbor Symposia on Quantitative Biology; 1989.

5. Unanue ER. Studies in listeriosis show the strong symbiosis between the innate cellular system and the T-cell response. Immunol Rev. 1997; 158(1):11-25.

6. Dempsey PW, Allison ME, Akkaraju S, Goodnow CC, Fearon DT. C3d of complement as a molecular adjuvant: bridging innate and acquired immunity. Science. 1996;271(5247):348-350.

7. Vella AT, McCormack JE, Linsley PS, Kappler JW, Marrack P. Lipopolysaccharide interferes with the induction of peripheral $\mathrm{T}$ cell death. Immunity. 1995;2(3):261-270.

8. Sun JC, Beilke JN, Lanier LL. Adaptive immune features of natural killer cells. Nature. 2009;457(7229):557-561.

9. O'Leary JG, Goodarzi M, Drayton DL, von Andrian UH. T cell-and B cell-independent adaptive immunity mediated by natural killer cells. Nat Immunol. 2006;7(5):507-516.

10. NOSSAL GJ. Molecular and cellular aspects of immunologic tolerance. Eur J Biochem. 1991;202(3):729-737.

11. Hoffmann JA, Kafatos FC, Janeway CA, Ezekowitz R. Phylogenetic perspectives in innate immunity. Science. 1999;284(5418):1313-1318

12. Hoebe K, Janssen E, Beutler B. The interface between innate and adaptive immunity. Nat Immunol. 2004;5(10):971-974.

13. Medzhitov R. Recognition of microorganisms and activation of the immune response. Nature. 2007;449(7164):819-826.

14. Bertrand N, Leroux J-C. The journey of a drug-carrier in the body: an anatomo-physiological perspective. J Control Release. 2012;161(2): $152-163$.

15. Storm G, Belliot SO, Daemen T, Lasic DD. Surface modification of nanoparticles to oppose uptake by the mononuclear phagocyte system. Adv Drug Deliv Rev. 1995;17(1):31-48.

16. Moghimi S, Gray T. A single dose of intravenously injected poloxaminecoated long-circulating particles triggers macrophage clearance of subsequent doses in rats. Clin Sci. 1997;93(Pt 4):371-379.

17. Dams ET, Laverman P, Oyen WJ, et al. Accelerated blood clearance and altered biodistribution of repeated injections of sterically stabilized liposomes. J Pharmacol Exp Ther. 2000;292(3):1071-1079.

18. Ishihara T, Maeda T, Sakamoto H, et al. Evasion of the accelerated blood clearance phenomenon by coating of nanoparticles with various hydrophilic polymers. Biomacromolecules. 2010;11(10): 2700-2706.

19. Suzuki T, Ichihara M, Hyodo K, et al. Accelerated blood clearance of PEGylated liposomes containing doxorubicin upon repeated administration to dogs. Int J Pharm. 2012;436(1):636-643.

20. Liu F, Liu D. Long-circulating emulsions (oil-in-water) as carriers for lipophilic drugs. Pharm Res. 1995;12(7):1060-1064.

21. Lundberg B, Mortimer B-C, Redgrave T. Submicron lipid emulsions containing amphipathic polyethylene glycol for use as drug-carriers with prolonged circulation time. Int J Pharm. 1996;134(1):119-127.

22. Hansrani PK, Davis SS, Groves MJ. The preparation and properties of sterile intravenous emulsions. J Parenter Sci Technol. 1983;37(4): $145-150$.

23. Wang C, Cheng X, Sui Y, et al. A noticeable phenomenon: Thiol terminal PEG enhances the immunogenicity of PEGylated emulsions injected intravenously or subcutaneously into rats. Eur J Pharm Biopharm. 2013;85(3):744-751. 
24. Bradley AP. The use of the area under the ROC curve in the evaluation of machine learning algorithms. Pattern Recogn. 1997;30(7): 1145-1159.

25. Rooijen NV, Sanders A. Liposome mediated depletion of macrophages: mechanism of action, preparation of liposomes and applications. J Immunol Methods. 1994;174(1):83-93.

26. Selye H. A syndrome produced by diverse nocuous agents. Nature. 1936;138(3479):32.

27. Weiss JM, Sundar SK, Becker KJ, Cierpial MA. Behavioral and neural influences on cellular immune responses: effects of stress and interleukin-1. J Clin Psychiatry. 1989;50 Suppl:43-53; discussion 54-55.

28. Koide H, Asai T, Hatanaka K, et al. T cell-independent B cell response is responsible for $\mathrm{ABC}$ phenomenon induced by repeated injection of PEGylated liposomes. Int J Pharm. 2010;392(1-2):218-223.

29. Ishida T, Wang X, Shimizu T, Nawata K, Kiwada H. PEGylated liposomes elicit an anti-PEG IgM response in a T cell-independent manner. J Control Release. 2007;122(3):349-355.

30. Cheng TL, Chen BM, Chern JW, Wu MF, Roffler SR. Efficient clearance of poly (ethylene glycol)-modified immunoenzyme with anti-PEG monoclonal antibody for prodrug cancer therapy. Bioconjug Chem. 2000;11(2):258-266.

31. Cheng TL, Wu PY, Wu MF, Chern JW, Roffler SR. Accelerated clearance of polyethylene glycol-modified proteins by anti-polyethylene glycol IgM. Bioconjug Chem. 1999;10(3):520-528.

32. Mebius RE, Kraal G. Structure and function of the spleen. Nat Rev Immunol. 2005;5(8):606-616.
33. Ishida T, Ichihara M, Wang X, Kiwada H. Spleen plays an important role in the induction of accelerated blood clearance of PEGylated liposomes. J Control Release. 2006;115(3):243-250.

34. Moghimi S, Patel H. Serum-mediated recognition of liposomes by phagocytic cells of the reticuloendothelial system-the concept of tissue specificity. Adv Drug Deliv Rev. 1998;32(1):45-60.

35. Rodrigueza WV, Phillips MC, Williams KJ. Structural and metabolic consequences of liposome-lipoprotein interactions. Adv Drug Deliv Rev. 1998;32(1):31-43.

36. Laverman P, Carstens MG, Boerman OC, et al. Factors affecting the accelerated blood clearance of polyethylene glycol-liposomes upon repeated injection. J Pharmacol Exp Ther. 2001;298(2):607-612.

37. Ichihara M, Shimizu T, Imoto A, et al. Anti-PEG IgM response against PEGylated liposomes in mice and rats. Pharmaceutics. 2010;3(1): $1-11$.

38. Cohen-Sela E, Rosenzweig O, Gao J, et al. Alendronate-loaded nanoparticles deplete monocytes and attenuate restenosis. J Control Release. 2006;113(1):23-30.

39. Epstein H, Berger V, Levi I, et al. Nanosuspensions of alendronate with gallium or gadolinium attenuate neointimal hyperplasia in rats. J Control Release. 2007;117(3):322-332.

40. Reiche EMV, Morimoto HK, Nunes SMV. Stress and depressioninduced immune dysfunction: implications for the development and progression of cancer. Int Rev Psychiatry. 2005;17(6):515-527.

41. Brown MR, Smith AW. Dormancy and persistence in chronic infection: role of the general stress response in resistance to chemotherapy. $J$ Antimicrob Chemother. 2001;48(1):141-142. 


\section{Supplementary materials}

\section{Study of the in vitro release of tocopheryl nicotinate (TN) from the PEGylated emulsions Method}

PEGylated emulsion (PE) samples $(1 \mathrm{~mL})$ and $0.5 \%$ sodium dodecyl sulfate (SDS, $1 \mathrm{~mL}$ ), enclosed in dialysis bags (cellulose membrane, molecular weight cutoff $100 \mathrm{kDa}$; Spectrum Laboratories, Inc., Rancho Dominguez, CA, USA), were incubated in $100 \mathrm{~mL}$ dialysis medium (pH 7.4 phosphate buffered saline (PBS) containing $0.25 \% \mathrm{SDS}$ ) at $37^{\circ} \mathrm{C} \pm 0.5^{\circ} \mathrm{C}$ under an agitation with $100 \mathrm{rpm}$ in a water bath. At 0.5 , 1.0, 2.0, 4.0, 6.0, 8.0, 12.0, and 24.0 hours, $2 \mathrm{~mL}$ samples were withdrawn from the incubation medium and analyzed for TN with high performance liquid chromatography as described in the Materials and methods. After sampling, the incubation medium was replaced by the dialysis medium. A control experiment to determine the release behavior of the free drug was performed. TN was dissolved in $0.25 \%$ SDS solution $(0.2 \mathrm{mg} / \mathrm{mL})$, and $2 \mathrm{~mL}$ of this solution was enclosed in a dialysis bag and immersed in $100 \mathrm{~mL}$ dialysis medium at $37^{\circ} \mathrm{C} \pm 0.5^{\circ} \mathrm{C}$. Then, the procedure described above for PE samples was followed.

\section{Results and discussion}

Before comparing the circulation time of $\mathrm{PE}$ in vivo, it was important to assess the release of TN from PE. The in vitro release curves of TN solution and TN from PE are shown in Figure S1. Free TN could completely permeate through the dialysis bag at 12 hours, while there was a relatively small amount of drug that leaked from PE. The cumulative release rate of TN from PE was $13.21 \% \pm 3.02 \%$ at 12 hours. The drug leakage from PE was significantly slower than that of TN solution. The result demonstrated that the majority of TN was dispersed in the medium-chain triglycerides. Therefore, the pharmacokinetics of $\mathrm{TN}$ could be a relatively true reflection of the in vivo behavior of PE.

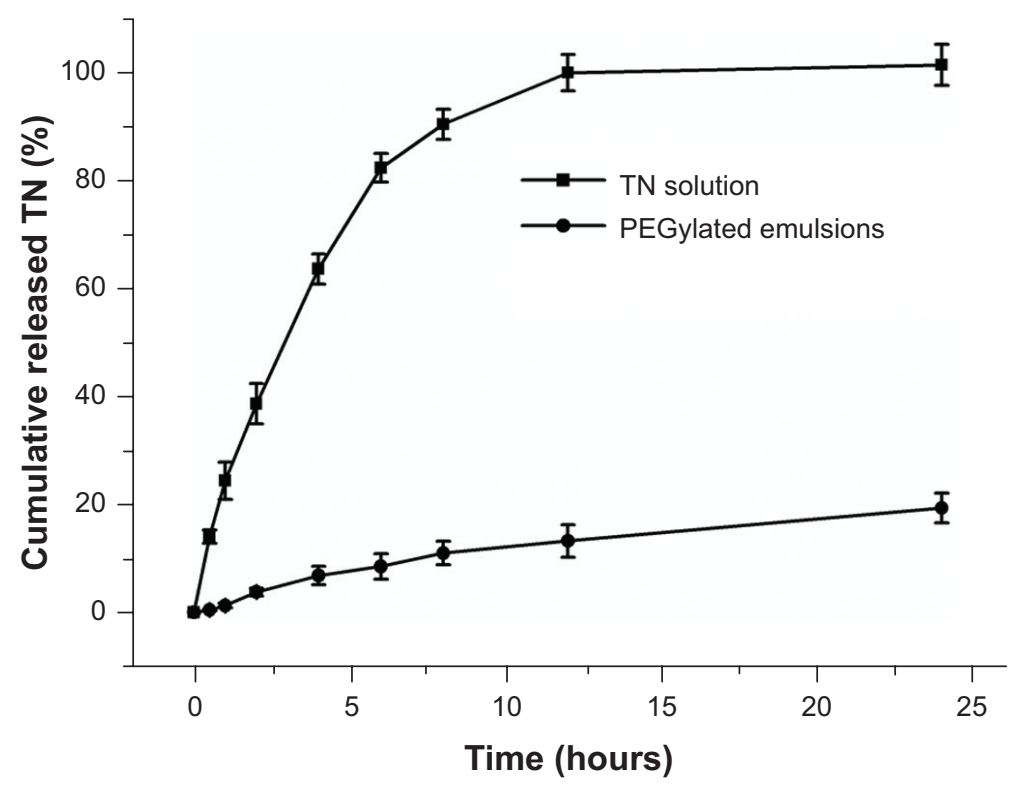

Figure SI In vitro TN release from PEGylated emulsions and TN solution in the PBS (pH 7.4) containing 0.25\% SDS.

Abbreviations: PBS, phosphate buffered saline; PEG, polyethylene glycol; SDS, sodium dodecyl sulfate; TN, tocopheryl nicotinate.

\section{Publish your work in this journal}

The International Journal of Nanomedicine is an international, peerreviewed journal focusing on the application of nanotechnology in diagnostics, therapeutics, and drug delivery systems throughout the biomedical field. This journal is indexed on PubMed Central,

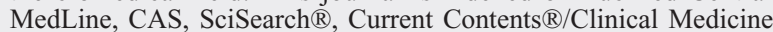

Journal Citation Reports/Science Edition, EMBase, Scopus and the Elsevier Bibliographic databases. The manuscript management system is completely online and includes a very quick and fair peer-review system, which is all easy to use. Visit http://www.dovepress.com/ testimonials.php to read real quotes from published authors. 